\title{
Medical Nutrition Therapy delays Dialysis and Improves Biomarkers
} de Waal $D^{*}$

University of Vermont Medical Center, USA

There is a high economic burden for kidney disease. Healthy People 2020 has a major goal of reducing new cases of kidney disease and its related complications, disability and early death [1]. The basic function of the kidney is the removal of waste products from the blood while regulating body water and electrolytes. As kidney disease progresses, altered nutrition biomarkers are observed which may be related to poor dietary habits [2]. Patients with kidney disease are often prescribed diets that are low in potassium and provided handouts that focus on limiting fruits and vegetables. Patients become confused and frustrated as this recommendation is at odds with prevention diets such as heart healthy and diabetes diets which emphasize fruits, vegetables and whole grains. The typical North American diet is high in protein and processed foods which can affect the balance of the body's electrolytes, minerals and contributes to the uremic environment of the digestive system. Evolving evidence has found a link between the gut and kidney health [3] suggesting a need for emphasis on nutrition for the care of a patient with compromised kidney function.

A poor diet is a major contributor to disease and early death [4]. Nutrition has become a mainstream social issue and the public's interest in nutrition and wellness has exploded [5]. In response, the Affordable Care Act supports preventative health services and has included access to wellness programs [6] including Medical Nutrition Therapy (MNT). The key practitioners of MNT are the registered dietitian (RD) and registered dietitian nutritionist (RDN). They use an evidenced-based application of the Nutrition Care Process which includes all domains of nutrition intervention including food and/or nutrient delivery, nutrition education, nutrition counseling, and coordination of nutrition care $[7,8]$. Nutrition and MNT is crucial in current and emerging health care models for wellness, health promotion, disease prevention, and disease management.

Given the exploding interest in nutrition and health, television, magazines and the internet have become popular sources of information [5]. Unfortunately, material on the internet often lacks editorial oversight and is replete with misinformation about nutrition and health. When faced with the prospect of kidney failure, patients are increasingly turning to alternative therapies. One of the fundamental principles of dietetic practice for an RD/RDN is to recommend nutrients from food sources as first line therapy. Supplements are only recommended if diet change is insufficient. RD/RDN's help coach patients make food and nutrient choices that are beneficial and help clear up any misconceptions. The public has acknowledged that the $\mathrm{RD} / \mathrm{RDNs}$ are a reliable source of reliable information on nutrition [5] who are the food and nutrition experts who can translate the science of nutrition and provide practical solutions for healthy living [8].

My renal nutrition clinic started in 2003 and over the years patients would comment that they felt the practical nutrition advice they received contributed in keeping them off dialysis longer than expected. This inspired me to do a 10 year retrospective study which was recently published [9]. Results demonstrated that the decline in kidney function was less in patients who received MNT at follow-up compared to those not receiving MNT. The participants who received MNT were less likely to start dialysis and had more favorable biomarkers. Albumin and biomarkers of chronic kidney disease- mineral and bone disorder were more likely to be within normal limits in the MNT group. Patients received individualized nutrition counselling with visits taking at least 60-90 minutes. The study did have some limitations but the results were very encouraging.

The kidney diet is one of the most challenging obstacles patients face. Diet is often entrenched as part of a person's lifestyle and they do not feel an immediate negative physical response with poor diet choices. An $\mathrm{RD} / \mathrm{RDN}$ with experience in kidney disease is uniquely qualified to coach patients with positive dietary choices that can help preserve their kidney function. The diet does not need to be an empty plate. Medicare has supported MNT provided by an RD/RDN for kidney disease since 2002 [10] but is presently underutilized. Strong evidence supports optimal nutritional status as the cornerstone in health maintenance [11]. With costs of kidney disease rising, it seems prudent to recommend a therapy that has been shown to delay the progression of kidney disease and improve biomarkers [9,11]. Medical Nutrition Therapy by an RD/RDN specializing in kidney disease should be recommended as one of the first therapies as soon as a medical diagnosis of kidney failure or even proteinuria has been made. Quality of life is dependent on the ability to make choices, and offering broader dietary choice provides patient empowerment contributing to greater enjoyment of life with a better nutrition status. MNT has the potential to improve quality of life of patients with kidney disease by improving their nutritional biomarkers and keeping them off dialysis longer.

"Let food be thy medicine and medicine be thy food." Hippocrates.

\section{References}

1. (2015) US Department of Health and Human Services. HealthyPeople.gov. Office of Disease Prevention and Health Promotion, Healthy People 2020.

2. Chan M, Kelly J, Batterham M, Tapsell (2014) A High Prevalence of Abnormal Nutrition Parameter Found in Predialysis End-Stage Kidney Disease: Is It a Result of Uremia or Poor Eating Habits? J Ren Nut 24: 292-302.

3. Rossi M, Johnson DW, Campbell KL (2015) The Kidney-Gut Axis: Implications for Nutrition Care. J Ren Nutr 5: 399-403.

4. Forouzanfar MH, Alexander L, Anderson HR, Bachman VF, Biryukov S, et al. (2015) Global, regional, and national comparative risk assessment of 79 behavioural, environmental and occupational, and metabolic risks or clusters of risks in 188 countries, 1990-2013: A systematic analysis for the global burden of disease study 2013

5. (2015) Nutrition and You: Trends 2011: Academy of Nutrition and Dietetics.

6. (2015) Medicaid.gov, Affordable Care Act.

*Corresponding author: Desiree de Waal MS RD CD FAND, Renal Dietitian/ Research Coordinator, University of Vermont Medical Center, Nephrology, 1 South Prospect Street, Burlington, VT, 05401, USA, Fax: (802) 847-3607; E-mail: Desiree.deWaal@UVMHealth.org

Received: December 02, 2015; Accepted: December 06, 2015; Published: December 14, 2015

Citation: de Waal D (2015) Medical Nutrition Therapy delays Dialysis and Improves Biomarkers. J Kidney 1: 106. doi:10.4172/2472-1220.1000106

Copyright: (c) 2015 de Waal D, et al. This is an open-access article distributed under the terms of the Creative Commons Attribution License, which permits unrestricted use, distribution, and reproduction in any medium, provided the original author and source are credited. 
7. (2015) Academy of Nutrition and Dietetics. Definition of Terms list.

8. (2015) Academy of Nutrition and Dietetics. Learn More about RDNs.

9. de Waal D, Heaslip E, Callas P (2016) Medical Nutrition Therapy for Chronic Kidney Disease Improves Biomarkers and Slows Time to Dialysis. J Ren Nutr 26:1-9.
10. (2015) Centers for Medicare \& Medicaid Services. National Coverage Determination (NCD) for Medical Nutrition Therapy.

11. (2015) Academy of Nutrition and Dietetics Evidence Analysis Library. 